\title{
Defect-assisted Reorganization of Ferroelectric Domain Walls Revealed by Aberration-corrected Electron Microscopy
}

\author{
Lin-Ze Li ${ }^{1}$, Jacob Jokisaari ${ }^{1}$, Yi Zhang ${ }^{1}$, Xiao-Xing Cheng ${ }^{2}$, Long-Qing Chen ${ }^{2}$, Colin Heikes ${ }^{3}$, Darrell \\ Schlom $^{3}$, and Xiao-Qing Pan ${ }^{1,4}$ \\ 1. Department of Chemical Engineering and Materials Science, University of California - Irvine, Irvine, \\ CA. \\ 2. Department of Materials Science and Engineering, Penn State University, University Park, PA. \\ 3. Department of Materials Science and Engineering, Cornell University, Ithaca, NY. \\ 4. Department of Physics and Astronomy, University of California - Irvine, Irvine, CA.
}

Domain walls (DWs) in ferroelectrics are quasi-2D functional units possessing peculiar properties that are absent in the bulk materials. The ability to produce ordered patterns of DWs is a prerequisite both for the fundamental study of DW properties and the design of novel nanodevices based on DW functionalites. In recent years, extensive efforts have been made towards fabricating periodic domain and DW structures in ferroelectric thin films, mainly through modifying elastic and electrostatic boundary conditions at the film interfaces. One of the major limitations of such a method, however, has been that once the choice of substrate is set further modifications to control or alter domain patterns during material synthesis becomes difficult, reducing the parameter space for creating more complex structures with ordered DW patterns and thus imposing resitrictions on the functionalities of the system. Here, with a combination of aberration-corrected scanning transmission electron microscopy (STEM) and electron energy loss spectroscopy (EELS), we show that atomically-thin charged defects that are deliberately introduced during the film growth can be used as nano-building-blocks for tailoring and reorganizing DW patterns. Such defect engineering can produce novel periodic mixed-type DW structures that are inaccessible by conventional boundary-condition-tuning methods

Epitaxial $\mathrm{BiFeO}_{3}$ films were grown on $\mathrm{TbScO}_{3}$ substrates by molecular-beam epitaxy. By tuning the substrate temperature during film growth [1], an array of atomically thin defects was introduced in the $\mathrm{BiFeO}_{3}$ matrix at a nearly constant level that is $110-130 \mathrm{~nm}$ above the bottom interface of the film. Fig. 1a shown by a bright-field transmission electron microscopy (TEM) image of the $\mathrm{BiFeO}_{3} / \mathrm{TbScO}_{3}$ heterostructure, with the resolved domain structures in the $\mathrm{BiFeO}_{3}$ film depicted schematically in Fig. 1b. Interestingly, a transformation from $109^{\circ}$ to $71^{\circ}$ domain patterns across the defects is observed, resulting the formation of novel mixed-type domains containing "head-to-head" positively charged DWs located exactly at the defects. As shown in a high-angle annular dark-field (HAADF) STEM image (Fig. $2 \mathrm{a}$ ), the defect is mostly composed of one type of stepped units (indicated by yellow arrows), which have an inclined orientation and are formed from one pair of $\mathrm{Bi}$ atoms alternating with two pairs of $\mathrm{Fe}$ atoms. The $\mathrm{O} \mathrm{K}$-edge electron energy loss near edges structures (ELNES) for the $\mathrm{BiFeO}_{3}$ matrix and the stepped defect are shown in Fig. 2b. The most evident difference between the two spectra is in the A peak region from 530 to $538 \mathrm{eV}$. While a clears plitting of the A peak into two subpeaks, A1 at $532 \mathrm{eV}$ and $\mathrm{A} 2$ at $535 \mathrm{eV}$, is observed in the spectrum of the $\mathrm{BiFeO}_{3}$ matrix, the absence of $\mathrm{A} 2$ in the spectrum of the defect results in a shape of the A peak that is more similar to $\gamma-\mathrm{Fe}_{2} \mathrm{O}_{3}$. The accumulation of Fe and $\mathrm{O}$ at the defects is further convinced by EELS spectrum images of a segment on the defect (Fig. 2c-e). With these $\gamma-\mathrm{Fe}_{2} \mathrm{O}_{3}$-like structures, an excess of anions (more oxygen) should accumulate at the defects and thus introduce net negative charge. Such charge accumulation can produce strong built-in fields leading to the observed reorganization of domain walls 
In conclusion, our results show that the introduced defects in $\mathrm{BiFeO}_{3}$ films are strongly coupled to the ferroelectric polarization, leading to a reorganization of domain-wall patterns across the defects. Since unique properties have been observed for different types of DWs, such ability to combine more than one periodic DW structure in a single film provides new opportunities both for studying the exotic properties of DWs, and is a novel, flexible design strategy for devices [2].

\section{References:}

[1] Li, L.Z. et al, Phys. Rev. Lett., (2018).

[2] The author gratefully acknowledges the financial support by the Department of Energy (DOE) under grant DESC0014430.
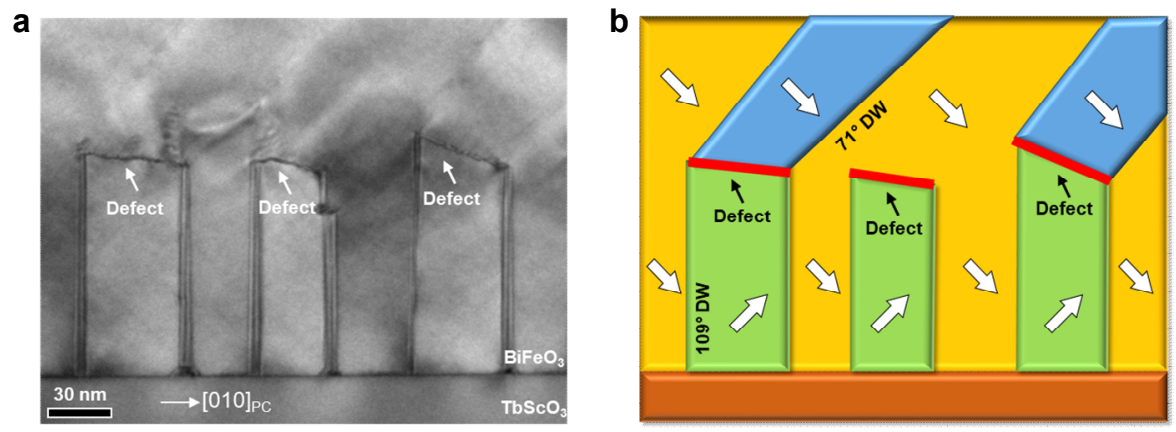

Figure 1. Defect induced domain-wall reorganization. (a) Cross-sectional bright-field TEM image and (b) corresponding schematic of polarization structures showing ordered $71^{\circ}$ and $109^{\circ}$ domains separated by an array of defects in a $400 \mathrm{~nm} \mathrm{BiFeO}_{3}$ film.

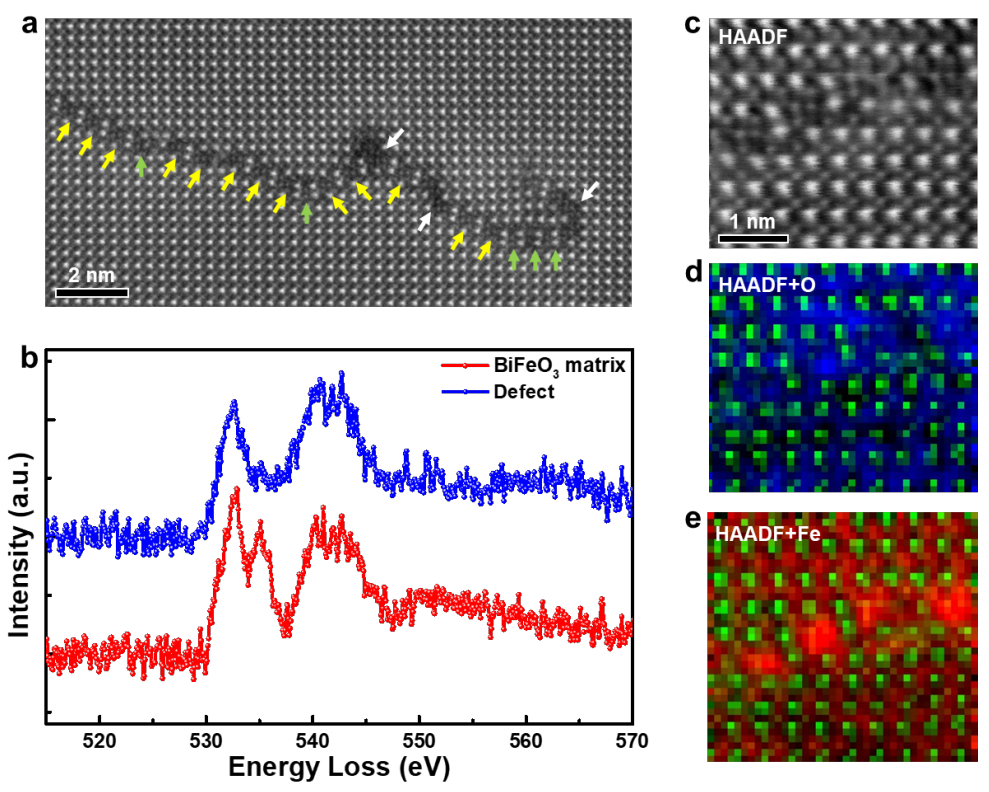

Figure 2. Atomic structures and chemical information at the defects. (a) Atomic-scale HAADF STEM image of a linear defect inserted in the $\mathrm{BiFeO}_{3}$ matrix. (b) O-K ELNES of the $\mathrm{BiFeO}_{3}$ matrix and the stepped defect. (c) HAADF STEM image of a segment on the defect and corresponding spectrum images of the same area: (d) combined map of $\mathrm{O}$ and HAADF signal (A-site atoms, i.e., $\mathrm{Bi}$ ) and (e) combined map of Fe and HAADF signal. 